\title{
Hacer Teatro: beneficios para el desarrollo positivo en adolescentes
}

\section{Acting: benefits for positive development in adolescents}

Tomás Motos-Teruel*

\section{RESUMEN}

Este estudio se centra en analizar las opiniones de los adolescentes sobre los beneficios que para ellos supone participar en experiencias teatrales. Se han identificado a partir de seis grupos foco, con una muestra de 53 participantes, de entre 12 y 20 años. Los resultados apuntan a que dichas prácticas: promueven habilidades personales; ofrecen una oportunidad para explorar y expresar sentimientos; proporcionan un contexto de apoyo y seguridad; posibilitan hacer amigos; incrementan habilidades comunicativas; proporcionan recursos para ser empleados en otros ámbitos y momentos de la vida; ayudan a formar la identidad y a mejorar la autoestima; desarrollan destrezas para entender a los otros, y suponen una oportunidad para evadirse de las situaciones negativas. Se discuten las limitaciones y se reconoce la necesidad de seguir investigando esta temática con otros participantes y otros diseños de investigación.

Palabras clave: teatro, actividades dramáticas, desarrollo positivo, adolescencia, grupos foco

\section{ABSTRACT}

This article presents the research carried out about the benefits that adolescents manifest regarding the experience of acting. These are identified from 6 focus groups, with a sample of 53 people, aged between 12 and 20 years. The qualitative results suggest that these practices can mean a significant help to positive personal development, and can be summarized in: to help to promote different personal resources; to offer an opportunity to explore and express feelings; to provide a context of support and security; to make friends; to enhance communicative skills; to provide resources to be employed in other areas and times in people's life; to affirm identity and to strengthen autonomy; to develop skills to understand others; and to provide opportunity to escape from negative situations. The need to investigate this topic with other participants and other research designs is recognized.

Key words: theatre, drama, positive development, young people, focus group

\section{Introducción}

¿Existe relación entre participar en actividades teatrales y el desarrollo positivo en la adolescencia? Para responder esta pregunta se han indagado las opiniones de adolescentes, insertos en el sistema educativo formal, sobre los efectos que la práctica de las actividades teatrales supone para su desarrollo personal. En este

\footnotetext{
* Profesor Titular (jubilado) del Departamento de Didáctica y Organización Escolar, Facultad de Filosofía y Ciencias de la Educación, Universitat de València, España; e-mail: tomas. motos@uv.es
} 
artículo $^{1}$ se presentan solo los resultados obtenidos mediante la metodología cualitativa con grupos foco.

Cuando se habla de teatro en la educación se suele distinguir entre teatro y drama. Se entiende por este la actividad en que los participantes están involucrados en un trabajo más espontáneo e improvisado y, por teatro, el actuar en un escenario. Ver y hacer teatro son los dos aspectos complementarios que se han de buscar en la educación artística. En el presente trabajo se ha colocado el foco en el hacer, ya sea en grupos de teatro extraescolar o en las actividades y contenidos teatrales de las asignaturas curriculares.

La variedad de actividades dramáticas es muy amplia. Entre otras: juego de expresión, juego dramático, dramatización, role play, improvisación, simulaciones, lectura de textos teatrales, reader's theatre, escritura individual y en grupo de textos dramáticos, escenificación de diálogos y escenas cortas, modalidades de Teatro del Oprimido, representación de espectáculos, teatro representado por actores diferentes al alumnado, etc. Los sujetos de nuestra investigación no necesariamente las practican todas, sino que depende del proyecto docente de su profesorado o sus animadores.

En España, la educación teatral, cuya presencia en los currículos oficiales es pequeña, gira principalmente en torno a actividades extracurriculares. En la Enseñanza Secundaria Obligatoria (ESO) los contenidos referidos al texto teatral se imparten en lengua y literatura, los de expresión corporal en educación física y las enseñanzas específicas de las artes escénicas en la de asignatura optativa Artes Escénicas y Danza, impartida en $4^{\circ}$ curso de la ESO. En el Bachillerato de Artes, se cursa la materia 'Artes Escénicas'.

Autores como O’Neill (1995), Neelands, Baldwin y Fleming (2003), Laferrière y Motos (2003) o Navarro (2013) concretan las virtualidades del teatro y el drama como instrumento eficaz para desarrollar aspectos de las competencia en comunicación lin-

${ }^{1}$ Los resultados expuestos en el presente artículo se enmarcan en el proyecto de investigación "El Teatro y los jóvenes", desarrollado durante 2014 y 2017 en el Máster en Teatro Aplicado de la Universidad de Valencia. Se pretende sondear el pensamiento de los jóvenes y adolescentes sobre el impacto y la huella dejados tras la experiencia de hacer teatro o acceder a los contenidos teatrales, contrastar esos efectos con el desarrollo positivo y, finalmente, compararlos con sujetos que no practican el teatro. 
güística, cultural y artística, social y ciudadana, aprender a aprender y autonomía e iniciativa personal.

A través del teatro los jóvenes representan sus percepciones de la realidad, pues la actuación teatral es tanto simbólica como reflexiva (Schechner, 1985). Las representaciones teatrales son emocionales, vívidas y basadas en la experiencia. La práctica teatral propone un espacio físico y, sobre todo, psicológico, en el que los jóvenes pueden reevaluar y vivir la realidad desde sus propias actitudes personales y las de los personajes que representan. En este sentido, el drama opera en lo que Heathcote (citado en Johnson y O'Neill, 1991: 130) llama zona de no-penalty. Esta autora asegura que "el teatro es la vida representada en una zona de no sanción. Parece, se asemeja, pero realmente no es”. En esta área sin punición, los participantes pueden probar experiencias, pero sin tener que vivirlas en sus consecuencias reales. Por otra parte, el teatro proporciona la oportunidad de explorar muchos 'posibles yoes', adoptando diferentes perspectivas y actitudes, comprometiéndose emocionalmente y pudiendo llegar a percibir las cosas de manera diferente. Abundando en este sentido, Davis afirma que "el drama ofrece una clase especial de "experiencia" y "vivir a través de" [...] Lo que de verdad es interesante es que mientras que el contexto y el papel son ficticios, las emociones sentidas pueden ser 'reales' y al mismo tiempo se distancian de lo real' (2015: 72). Este estado de pertenecer simultáneamente a dos mundos diferentes y autónomos (el real y el del drama) es llamado por Boal (2004) metaxis.

Las actividades teatrales son liminares porque vienen calificadas por la libertad de expresión, por un espacio no institucionalizado y por la oportunidad de explorar o reinventar la propia identidad (Kim, 2015: 72). Se practican, pues, en un espacio transicional. Turner (2001) propone que el teatro es único en su capacidad de crear un contexto social que facilite la liminalidad, pues posibilita a los individuos residir entre los umbrales de lo cotidiano y lo imaginario, entre la realidad y la posibilidad. Como arte escénico, el teatro ha sido ampliamente celebrado por su capacidad para facilitar experiencias liminales, generando un espacio donde las normas y jerarquías previamente consolidadas 
pueden ser suspendidas, y a menudo invertidas, creando así oportunidades para el cambio personal y colectivo.

Beare y Belliveau (2007) han propuesto un modelo de desarrollo de las artes escénicas que comprende cinco etapas. En la primera (inclusion) los participantes pretenden integrarse en el grupo y pertenecer a él. En la segunda (control) la principal preocupación es saber si lo que hacen o dicen interesa a sus compañeros y si su habilidades teatrales se corresponden con lo que el grupo espera. En la tercera (intimacy) se pone el foco en establecer amistades y vínculos profundos. Quienes alcanzan la cuarta etapa (empowermen) son competentes y admirados, son conscientes de que los más jóvenes les emulan, y han de comportarse como sus modelos. Los que logran alcanzar la última etapa (vision) tienden a ser los líderes naturales del grupo debido a su profunda percepción del trabajo colaborativo y a sus habilidades para relacionarse con los demás. La mayor parte de los jóvenes que participan en actividades teatrales están una de las tres primeras etapas.

La adolescencia es el periodo de transición entre la infancia y la adultez, en el que se produce un proceso de maduración que implica cambios físicos, psicológicos y sociales (Alsinet, Perez y Agullo, 2003 y Stassen, 1997). Respecto a su rango de duración, aunque varía según las diferentes fuentes médicas, antropológicas y psicológicas, se suele admitir el inicio entre los 10 y 12 años y la finalización a los 19 o 24 (Organización Mundial de la Salud [OMS], 1986). En la adolescencia se produce una confusión y una crisis de identidad (Erikson, 1968), pues no se tienen convicciones formadas sobre "lo que soy ni sobre lo que quiero ser". En este sentido, los adolescentes suelen presentar algunas manifestaciones preocupantes desde el punto de vista de los adultos: cambios en su aspecto físico, conflictos con los padres y profesorado, inestabilidad emocional, bajo rendimiento escolar o conductas de riesgo como ejercicio precoz de la sexualidad, consumo de drogas, trastornos alimentarios, comportamientos delictivos e intentos de suicidio (Jiménez, Musitu y Murgui, 2005).

Para prevenir tales comportamientos de riesgo, actualmente se proponen patrones de intervención como el centrado en el déficit (Oliva, 2010), que considera la ausencia de problemas como indicador de un desarrollo sano. Estos nuevos enfoques siguen 
los pasos del modelo del desarrollo positivo y la competencia (Benson, Scales, Hamilton y Sesman, 2006). El denominado Positive Youth Development está enfocado al bienestar, pone el acento en la existencia de condiciones saludables y expande el concepto de salud incluyendo las habilidades, conductas y competencias necesarias para tener éxito en la vida social, académica y profesional (Benson, Mannes, Pittman y Ferber, 2004). Así, un adolescente saludable es aquel que no se implica en actividades antisociales (violencia, consumo de drogas o prácticas sexuales de riesgo, etcétera).

Una de las tareas centrales de la adolescencia es la búsqueda de la identidad personal y la construcción de una imagen coherente de sí mismo, que siente las bases para afrontar los desafíos de la etapa adulta, pues la identidad es una reflexión para la adaptación individual al contexto. Autores como Erikson (1983), Marcia (1980) o Kroger, (2004) han teorizado sobre la identidad del joven. Marcia la define como "una organización interna, autoconstruida y dinámica de impulsos, habilidades, creencias e historia individual" (1980: 159).

La adolescencia es un periodo especialmente oportuno para el desarrollo de la identidad (Zacarés, Iborra, Tomás y Serra, (2009), pues esta se fija al final de la adolescencia. Los adolescentes deben resolver la crisis de identidad versus la confusión de roles. Durante la adolescencia se produce un proceso de búsqueda interior, se cambian las características que definieron el yo en la nińez, se cuestionan los valores personales y aparecen nuevos compromisos y prioridades (Berk, 2004). La identidad consiste en el hecho de que alguien se reconozca a sí mismo. El yo es lo que identifica a cada persona y la hace diferente a los demás. Es nuestra identidad personal. Se caracteriza por su naturaleza múltiple y dinámica y por ser un espacio de negociación, nunca neutro, sino atravesado por diferentes relaciones de poder que conforman las subjetividades. Para Erikson (1983) la identidad implica un sentido de continuidad y coherencia del yo a lo largo de tiempo. Es decir, ser la misma persona del pasado que se recuerda, del presente que se vive y del futuro que se espera.

Para el presente estudio entenderemos por autoestima la valoración que la persona hace de sí misma. Constituye el elemento 
valorativo del autoconcepto, del autoconocimiento (Garaygordobil y Durá, 2006). La contribución de la autoestima sería la de proporcionar seguridad al sujeto a la hora de asumir riesgos y considerar opciones (Zacarés, Iborra, Tomás y Serra, (2009). En los adolescentes la autoestima crea una visión que influye en la forma en que se relacionan con las personas que les rodean y en cómo se sienten en relación con los demás.

Los datos disponibles indican que participar en programas de desarrollo positivo durante la adolescencia está relacionado con un mayor compromiso social durante la adultez temprana así como con mayores logros a nivel educativo y ocupacional (Gardner, Roth y Brooks-Gunn, 2008). Existen muchos trabajos que documentan la acción protectora de las actividades extracurriculares contra la implicación en actos delictivos y conductas problemáticas (Persson, Kerr y Stattin, 2007). Larson et al. (2004) señalan que las actividades extraescolares tienen una serie de consecuencias en el desarrollo positivo del adolescente: mejora de la iniciativa personal, aumento de la motivación, establecimiento de relaciones interpersonales incluyendo a personas adultas, aceptación de la diferencia y fortalecimiento de la personalidad. Igualmente, están documentadas ventajas a nivel de relaciones interpersonales, como una mayor capacidad de iniciativa y de toma de decisiones, mejor manejo de los conflictos, mayores habilidades de comunicación y, en general, más habilidades sociales (Eccles et al. 2003). Los estudios longitudinales parecen confirmar la relación entre participar en actividades y el desarrollo positivo a lo largo del tiempo (Gardner, Roth y Brooks-Gunn, 2008).

Lerner et al. (2011) han realizado un estudio longitudinal (4-H Study of Positive Youth Development-PYD-) durante siete años, con 7000 adolescentes, para poner a prueba la hipótesis de que cuando las fortalezas de los jóvenes están alineadas con los recursos de la familia, la escuela y la comunidad, se producirá un desarrollo positivo. El programa, denominado 5C y diseñado para confirmar esta idea, está integrado por cinco áreas de intervención: competence (visión positiva de las propias acciones, asertividad, habilidades de resolución de conflictos y toma de decisiones); connection (establecimiento de lazos positivos consigo, la comunidad, la escuela y los pares); confidence (sentido interno 
de autoestima y autoeficiencia); caring (simpatía, empatía e identificación con los demás) y character (respeto a las normas sociales y culturales, sentido de lo correcto y lo incorrecto). Las 5C conducen a una sexta: contribution (aportaciones a uno mismo, a la familia, a la comunidad y a la sociedad).

Puesto que uno de los ámbitos del teatro para hacer son las actividades extracurriculares, es fácilmente deducible que los efectos señalados son también aplicables a las actividades teatrales. Los investigaciones realizadas, en el ámbito anglosajón (Dutton, 2001; Hughes y Kim et al., 2015 y Wilson, 2004) coinciden en el impacto en el desarrollo personal y social de los jóvenes y en que tiene efectos positivos sobre la promoción de recursos personales útiles para resolver de forma adecuada los retos vitales que se plantean en la adolescencia.

Dutton (2001) desarrolló un proyecto basado en experiencias dramáticas para trabajar con adolescentes en la construcción de competencias, el fortalecimiento de la autoestima y el fomento del reconocimiento de virtudes y fortalezas personales. Hughes y Wilson (2004) trabajaron con trescientos jóvenes y concluyeron que el teatro les ayudaba a desarrollar su sentido de identidad, su iniciativa, a tomar riesgos y a mejorar su habilidad para expresarse. Daykin et al. (2008) realizaron un metaestudio sobre las consecuencias de participar en actividades teatrales sobre la salud y el comportamiento de jóvenes universitarios. Concluyen que existen suficientes evidencias para afirmar que dicha práctica tiene efectos positivos en capacidades tales como habilidades sociales, empoderamiento, actitudes y comportamientos de riesgo (prácticas sexuales, alcohol, tabaco y consumo de drogas).

En el ámbito hispanófono no existen estudios empíricos sobre el efecto que tiene hacer teatro en los adolescentes, a excepción del de Barquero y Vargas (2009), que investigaron los efectos de la improvisación teatral y la expresión corporal con jóvenes universitarios y concluyeron que tiene efectos positivos y significativos sobre el estado de ánimo de los participantes así como en su autoestima y confianza. Por esta razón, hemos intentado comprobar empíricamente si los resultados de los estudios anteriores sobre los efectos de las actividades teatrales en el desarrollo positivo se producen también en los adolescentes españoles. 
Para nuestra investigación hemos tomado como elementos definidores del desarrollo positivo las cinco competencias de Lerner et al. (2011) a las que hemos ańadido seis de las competencias específicas que considera Oliva (2010: 230): autoestima, habilidades sociales, reconocimiento y manejo de las propias emociones y las de otras personas, pertenencia y vinculación, habilidades comunicativas y compromiso. Puesto que en nuestra investigación nos preguntamos si existe relación entre participar en actividades teatrales y el desarrollo positivo de los adolescentes, esta cuestión nos permite plantear las siguientes hipótesis:

1. Los adolescentes españoles valoran de forma favorable la influencia de la experiencia teatral sobre su desarrollo personal.

2. Las opiniones manifestadas por los adolescentes españoles sobre la vivencia y el impacto de la actividad teatral giran en torno a dimensiones tales como: expresión y gestión de sentimientos; sentido de compromiso; sentido de pertenencia, apoyo y aceptación; evasión de los problemas personales; aumento y mejora de las habilidades sociales; habilidades comunicativas y transferencia de lo aprendido en teatro al contexto escolar y familiar.

3. La identidad y la autoestima son las dimensiones más destacadas en la relación entre actividades teatrales y el desarrollo personal positivo.

\section{Método}

El presente estudio se centra en la exploración de las declaraciones y valoraciones que los jóvenes manifiestan tras vivir la experiencia de la práctica del teatro, entendida como teatro para hacer.

Para tratar de responder la pregunta planteada preparamos un diseño de investigación no experimental, transversal y descriptivo. Dada la naturaleza de los resultados que se pretendía obtener era necesaria una metodología cualitativa, pues nuestra investigación se basa en el análisis subjetivo e individual de aquellos. Se trata, pues, de una investigación interpretativa. 


\section{Muestra}

Los sujetos que participaron fueron 53, distribuidos en cinco grupos de discusión. De ellos, dos se realizaron en los II Encuentros de Teatro en la Educación, celebrados en Valencia, entre el 12 y el 16 mayo de 2014; y tres, en los III Encuentros durante el 20 y el 24 de abril de 2015. Los participantes procedían de 15 institutos de la ciudad de Valencia y de pueblos del área de influencia, 12 públicos y tres privados concertados. El rango de edad: 12-20 años, la moda:16, el promedio:15, 6 y la desviación estándar: 1,69. En cuanto a la distribución por género, 38 chicas y 15 chicos. Los estudios que cursaban eran $2^{\circ}, 3^{\circ}, 4^{\circ}$ de ESO, $1^{\circ}$ y $2^{\circ}$ de Bachillerato y Formación Profesional de Grado Medio. Las modalidades: optativa de Taller de Teatro en ESO, Bachillerato Artístico, taller de teatro extraescolar y Técnico en Animación Sociocultural.

La selección de los participantes fue intencional o de conveniencia, puesto que elegimos individuos a los que se tenía fácil acceso, utilizando como criterio el que estuvieran vinculados con el estudio y la práctica del teatro, en alguna de las modalidades citadas.

\section{Instrumento y procedimiento}

La técnica de investigación utilizada ha sido el análisis de contenido de las declaraciones de los participantes, recogidas durante los grupos de discusión. Estos constituyen una técnica de recogida de datos de naturaleza cualitativa, ampliamente empleada en diferentes campos de la investigación sociológica.

El tipo de grupo de discusión fue cara a cara. El tamaño de los grupos osciló entre 15 y 8 participantes (3, de 8 ; 1 de 14 y 1 , de 15). Con cada uno se preparó una sesión que duró 1:30 horas.

Entre las actividades de los encuentros había diferentes talleres. Y, como una actividad más de las programadas, estaban los grupos de discusión, en los que participaron chicos y chicas elegidos al azar.

El moderador de las sesiones fue el investigador, cuya función fue estimular la discusión a través de preguntas abiertas, reencau- 
zar el debate cuando los participantes se dispersaban del tópico en cuestión y evitar el predominio de algunos invitando a hablar a los más silenciosos.

Tras los saludos y presentación, se les informó del propósito de la investigación y se obtuvo su consentimiento verbal para grabar las sesiones y, posteriormente, transcribirlas y hacer uso de sus intervenciones. Ahora bien, no se les propuso que firmaran individualmente ningún impreso de consentimiento. Consideramos que se han respetado los requisitos mínimos de la ética.

Se les comunicó que cada participante podía intervenir libremente no solo respondiendo a lo que el moderador plantease, sino que cuando creyeran conveniente podían completar, profundizar o ampliar lo dicho por otros compañeros. Los ejes objeto de discusión fueron:

1. ¿Qué es el teatro para ti? Razones por las que participas en actividades teatrales. Tus primeros contactos con el teatro y quién te inició en las actividades teatrales.

2. Beneficios personales y mejoras que crees haber obtenido mediante el teatro.

3. ¿Hacer teatro ayuda a expresar y gestionar mejor los sentimientos?

4. ¿Con quién te sientes comprometido cuando preparas una representación o una dramatización?

5. ¿Participar en actividades teatrales ayuda a conocerse mejor? ¿Y a los demás? ¿Qué has descubierto de ti, que antes desconocías?

6. ¿Por quién te sientes apoyado? ¿Profesores, compañeros, padres? ¿Qué ambiente hay en tu grupo de teatro o en las clases de teatro de tu instituto?

7. ¿El teatro ayuda a evadirse de los problemas personales, familiares, con los compañeros, etcétera?

8. ¿El teatro mejora las relaciones con los compañeros?

9. ¿Lo que aprendes en el teatro lo pones en práctica en tu clase, con tus compañeros, en tu casa, en tu barrio?

Como espacio físico se dispuso del hall de la Sala Inestable, la organizadora de los eventos. El instrumento de grabación utiliza- 
do fue el teléfono móvil y, posteriormente, los audios se transcribieron. No se tuvo en cuenta ni fue tema de análisis el lenguaje corporal.

Las transcripciones fueron sometidos a análisis de contenido del discurso. Para el proceso de codificación de las transcripciones se utilizó el programa informático Atlas ti 7, herramienta computacional para el tratamiento y análisis cualitativo de grandes cuerpos de datos textuales, videos y gráficos.

No existe ninguna fórmula para identificar los temas (unidades de análisis) y agrupar estos en subcategorías y categorías Taylor y Bogdan recomiendan que hay que aprender a buscar temas examinado los datos de todos los modos posibles (1986: 160).

Las unidades temáticas o de análisis son "los segmentos que interesan investigar del contenido de los mensajes escritos susceptibles, posteriormente, de ser expresados en categorías y subcategorías [...]; son bases de sentido localizables en un texto" (Fernández, 2002: 38). Estos fragmentos de texto, frases o incluso palabras transmiten un contenido semántico relacionado con una pregunta estímulo formulada por el moderador. La familia o categoría es una etiqueta que engloba a todos aquellos elementos aparecidos en el discurso que tienen rasgos semánticos comunes. Por su parte, las subcategorías concretan y limitan el significado de las categorías. En nuestro caso, se procuró que fueran pertinentes, exhaustivas, homogéneas y mutuamente excluyentes.

\section{Resultados}

Los documentos primarios que utilizamos fueron las transcripciones de los discursos recogidos en las cinco sesiones de los grupos foco. A continuación se procedió a la codificación, asignando cada unidad de análisis a una subcategoría y estas, a su vez, a una categoría, identificadas ambas por sus respectivos códigos. Seguidamente, se realizó la cuantificación mediante el conteo de registros de cada categoría (ver cuadro 1) y. finalmente, se procedió a enlazar las subcategorías para diseñar las redes semánticas (ver figura 1). Los datos resultantes fueron tratados mediante el análisis estadístico descriptivo expresado en frecuencias y porcentajes. 
CUADR01. Categorización y porcentajes. Elaboración propia

\begin{tabular}{|c|c|c|c|}
\hline Categorías & Subcategorías & Unidades temáticas & Porcentaje \\
\hline \multirow[t]{4}{*}{ Beneficios } & $C P B$ & 209 & 19.56 \\
\hline & EV & 41 & 3.84 \\
\hline & TRV & 62 & 5.80 \\
\hline & Total & 312 & 29,21 \\
\hline \multirow{5}{*}{ Identificación } & PRE & 52 & 4.86 \\
\hline & ED & 52 & 4.86 \\
\hline & INS & 42 & 3.93 \\
\hline & THT & 35 & 3.27 \\
\hline & Total & 181 & 16.92 \\
\hline \multirow[t]{3}{*}{ Inicios } & ALT & 23 & 2.15 \\
\hline & INT & 29 & 2.71 \\
\hline & Total & 52 & 4.76 \\
\hline \multirow[t]{3}{*}{ Pensamiento } & RHT & 62 & 5.80 \\
\hline & PT & 91 & 8.52 \\
\hline & Total & 153 & 14.33 \\
\hline \multirow[t]{3}{*}{ Desafío } & RTR & 70 & 6.55 \\
\hline & $\mathrm{COM}$ & 53 & 4.96 \\
\hline & Total & 123 & 11.52 \\
\hline \multirow[t]{3}{*}{ Autoconciecia emocional } & SEG & 40 & 3.75 \\
\hline & EXS & 67 & 6.27 \\
\hline & Total & 107 & 10.02 \\
\hline \multirow[t]{4}{*}{ Relaciones personales } & RAM & 60 & 5.62 \\
\hline & APY & 80 & 7.49 \\
\hline & Total & 140 & 13.11 \\
\hline & TOTAL & 1068 & 100 \\
\hline
\end{tabular}

$\mathrm{CPB}=$ Cambio personal, EV = Evasión, TRV = Transferencia, $\mathrm{PRE}=$ Presentación, ED =Edad, INS= Instituto, $\mathrm{THT}=$ Tiempo haciendo teatro, ALT = Aprendizaje del lenguaje teatral, INT = Inicio de la experiencia teatral, $\mathrm{RTH}=$ Razones para hacer teatro, PT = Idea sobre el teatro, $\mathrm{RTR}=$ Retos y riesgos, $\mathrm{COM}=$ Compromiso, $\mathrm{SEG}=$ Seguridad, EXS $=$ Expresión de sentimientos, RAM $=$ Relaciones de amistad, APY $=$ Apoyo.

\section{Beneficios}

Esta categoría, que alcanza casi el 30\% de las unidades temáticas, se refiere a las mejoras percibidas y atribuidas a las actividades 
teatrales; a la transferencia de lo aprendido en teatro a la vida escolar y social; y a la percepción de ellas como un espacio de evasión de los problemas personales.

Dentro de esta categoría destaca la subcategoría Cambio personal (CPB) con aproximadamente una de cada cinco de las unidades temáticas. Es la más importante de todas, no solo por el número total de unidades que comparten un significado común sino también por la centralidad que ocupar en el mapa de relaciones semánticas (ver figura 1). Es la causa de las razones para hacer teatro y está asociada con el pensamiento sobre el teatro, la expresión de sentimientos, el sentimiento de seguridad, la evasión, el compromiso y la transferencia.

En las declaraciones de los participantes que intercalamos, las citas están identificadas con un nombre ficticio y un número, que indica la edad real del autor de las mismas.

Abundan las referencias a la mejora de la autoestima. Ejemplos:

Yo antes era una persona con la autoestima bastante baja y ahora, gracias al teatro, sinceramente, me ayudó a subírmela. (Marta15). Pues yo el año pasado era una persona totalmente distinta, era insegura, tenía muy poca autoestima, muy poca confianza, ahora todo ha cambiado. Ahora creo que me enorgullezco de lo que soy y antes [...] siempre pensaba que era lo peor (Sara15).

Pero también señalan otros beneficios:

Puedo utilizar mi imaginación cuando quiera. Pocas veces podía utilizarla y ahora siento que todo fluye (Ainhoa14). Aprendí a superar mis miedos (Marta14). Abrir la mente (Laura15). Tener vocabulario (Nadia14). Escuchar (Roberto15). Lo que más hemos reforzado ha sido la espontaneidad (Soraya16). He aprendido a respetar a los demás y a estar orgullosa de formarme día a día (Lourdes16). Yo he sido siempre un poco irresponsable, en el sentido de que he ido siempre a mi bola [...] Desde que empecé el teatro [...] me di cuenta de que cogí más responsabilidad, tanto dentro como fuera del escenario. Y yo creo que la gente que me conoce lo ha notado bastante (Sara15). 
Algunos participantes incluso cuantifican lo que han aprendido en el teatro.

El 70\% de todo. Aprender a respetar el punto de vista de los demás, saber comunicarte con los demás con respeto (Álvaro16). Para mí el 95\% porque es toda mi vida yo creo que [el teatro] me lo ha dado todo [...] Realmente donde yo creo que he aprendido de verdad, [...] es en el teatro (Carla17).

De sus declaraciones se deduce que para los participantes el teatro es una herramienta para la construcción de la identidad. Así lo manifiestan:

Siento que he crecido como persona (Lucía16). Creo que me ha ayudado a ser mejor persona (Josemi19). Yo no me atrevía a hablar con nadie. Y no era capaz de hacer amigos. Me costaba todo mucho. $Y$ ahora, pues soy yo misma (Andrea14). Yo empecé a ser persona con el teatro (Álvaro20). Dejas de ser un producto como te hace la sociedad para de verdad ser más [lo que] tú quieres ser. No como los otros quieren que seas (Marta17). Lo que más miedo me daba era que en mi casa no aceptaran mi homosexualidad [...] Pero ahora he ganado seguridad y me da igual lo que piensen de mí (Érica17).

Destacan perder la vergüenza a actuar, hablar o hacer alguna actividad en público, lo que conduce a los participantes declararse más desinhibidos abiertos y comunicativos.

Perder un poco la vergüenza y aprender a hablar en público. Pues yo cuando tenía que hacer trabajos delante de gente, [...] no me atrevía a hablar, porque yo era muy vergonzosa. Y ahora pues me da igual (Laura14).

La superación de la vergüenza hace que los participantes se sientan más.

Me acuerdo de que en el colegio siempre me decían que no conocían mi voz. Nunca hablaba. Y fue a partir de cuando me apunté a teatro que los profesores me decían has empezado a hablar. Porque era muy tímida (Sara14). El teatro me ha ayudado a ser menos tímido. Normalmente yo 
era una persona que me costaba relacionarme con los demás. Me costaba decir las cosas que pensaba de otros. Y ahora mismo gracias al teatro, soy más extrovertido (Roberto15). Era muy fría. A no ser que fuera gente que conocía, no quería que se me acercasen. El teatro ha hecho ser más abierta con la gente (Laura15).

La segunda subcategoría, con el $5.8 \%$, es Transferencia (TRV). Se refiere a que tomar parte en actividades teatrales de alguna forma proporciona habilidades y recursos que luego pueden ser empleados en otros sitios y momentos de la vida.

Lo que aprendemos en teatro es un aprendizaje continuo, se lleva de la escena a la calle ( Pau18). Te ayuda a expresarte mejor [...] Gracias al teatro, tengo aptitudes sociales desarrolladas y sé hablar y sé comunicarme con la gente. (Cristina16).

Lo que aprendemos en teatro es algo que utilizamos directamente en nuestra vida social. ( Iván15).

La tercera subcategoría, con un 3,84\%, es Evasión (EV). El teatro es un espacio para olvidar y escapar por unas horas de los problemas personales. Así lo declaran los participantes:

Llega a ser una vía para desahogarte. A evadirte de los problemas y de tu vida por un momento. Llegas a necesitarlo. Es una dependencia (Claudia 17). Yo tengo ganas de empezar teatro, porque es entrar y decir "adiós problemas", porque es un sitio donde disfrutas, sonríes, te lo pasas bien. Es como que los problemas se van disolviendo, se van diluyendo (Soraya16). A mí este año el teatro me ha servido para relajarme. Porque es un año complicadillo ya que segundo de bachillerato no se lleva bien. Y estar en teatro es un momento para olvidarme de la presión que tengo (Nadien18).

\section{Identificación}

La segunda categoría en cuanto a unidades temáticas (16.92\%) recoge los datos de personalización de los participantes y agrupa cuatro subcategorías. Presentación (PRE), reúne el 4.86\% de las 
unidades temáticas. Al comienzo de las sesiones se pedía a los participantes que dijeran su nombre, edad y el centro en el que estudiaban. Los datos referidos a Edad (ED) e Instituto (INS) están recogidos en la descripción de la muestra. Tiempo haciendo teatro (THT): algunos participantes lo precisan con exactitud y va desde los nueve meses (diez sujetos) a los nueve ańos (tres sujetos). Otros se limitan a expresarlo de una forma más imprecisa: "desde pequeña", "he estado desde los seis años hasta ahora y no he podido dejármelo", "yo llevo muy poco tiempo".

\section{Inicios.}

Es la categoría menos relevante pues solo recoge $4.76 \%$ de las unidades temáticas. Se refiere al inicio de la experiencia teatral ya sea en el contexto familiar, escolar o en el marco de la formación académica; y al conocimiento del leguaje dramático. Razones iniciación al teatro (INT): esta subcategoría, con un porcentaje de $2.71 \%$, detalla los motivos explicitados por los que el participante empieza a formar parte de un taller de teatro extraescolar o elige la asignatura optativa de Taller de teatro. Entre las razones de participar en actividades teatrales encontramos la importancia del grupo de iguales.

Yo empecé en teatro porque vas con un compañero de clase a una extraescolar y te apuntas para pasar el rato. Pero con los años se ha ido convirtiendo ya no en una actividad que me guste, sino en una actividad que necesito hacer para yo sentirme completo. Porque necesito el teatro, es por eso por lo que voy a teatro (Jesús20).

Yo me apunté porque como normalmente faltan chicos en el grupo... y me dijeron, "venga Diego, apúntate...", y por eso me apunté (Diego14).

Aprendizaje del lenguaje teatral (ALT). Subcategoría con solo $2.15 \%$ se refiere al aprendizaje de conocimientos y procedimientos propios del lenguaje teatral. Únicamente los participantes destacan los utilizados en su clase o taller: improvisación, expresión corporal y dramatización. 


\section{Pensamiento sobre el teatro}

Esta categoría, que supone $14.33 \%$, recoge las unidades de significado referidas a la idea que los participantes tienen sobre qué es teatro y las razones por las hacen actividades teatrales. La subcategoría Idea sobre el teatro (PT) agrupa no solo lo que los participantes piensan acerca de qué cosa sea el teatro, sino también cómo ha de ser el teatro para jóvenes. Y así declaran:

Es como un arma pacífica (Jesús20). Meterte en la vida de otras personas y tener como otra vida (Alejandro16). Olvidar quien eres y representar a otros (Laura14).

Me parece una cosa muy difícil, muy complicada pero a la par me parece también una cosa maravillosa y es un modo de vida (Marta14).

El teatro para los jóvenes ha de tener algunos de estos rasgos: 'un lenguaje que te atraiga', 'divertido, que haga reír', 'esté planteado de una forma que nos interese', 'con cambio de situaciones', 'trama sea fácil de seguir' y 'suspense'. Y en cuanto a los temas: 'la adolescencia', el amor', 'los estudios' o 'los conflictos cotidianos'.

Y respecto a los géneros y puesta en escena, hay para todos los gustos: la tragedia, la comedia, y que impacte por el tema y por la música, escenografía y uso de las nuevas tecnologías.

En cuanto a las Razones para hacer teatro (RHT), subcategoría con $5.80 \%$, he aquí algunas declaraciones:

Con los años se ha ido convirtiendo [...] en una actividad que necesito hacer para sentirme completo. Porque necesito el teatro, es por eso por lo que voy a teatro. (Álvaro20). Te sientes bien. Haces cosas que te gustan. Y cuando acabas, estás contenta, estás satisfecha con lo que has hecho. (Silvia16).

Entre las razones de hacer teatro está la de entrar en otros mundos y "vivir otros yoes". En este sentido, los sujetos declaran: 
Cuando haces teatro es como que te sales de tu mundo y puedes ser lo que tú quieras (Roberto15). Me encanta porque cuando salgo al escenario me olvido de todo y además de que puedo ponerme en la piel de otras personas, me hace, también, enseñar mis sentimientos, que es algo que no me gusta mucho. Entonces me olvido de que soy yo y me meto en el papel de otra persona y todo es mejor (Carles17).

\section{Desafíos}

Con $11.52 \%$ de las unidades temáticas hace referencia a los desafíos que supone el participar en actividades teatrales y el compromiso que ello conlleva. La subcategoría Retos y riesgos (RTR) representa $6.55 \%$ del total. Y se refiere a que hacer teatro y participar en las actividades de dramatización supone correr riesgos y responder a desafíos. Los principales de que tienen conciencia los sujetos de la muestra son cómo hacer llegar claramente al público el mensaje de la obra, atraer su atención, mantenerla y que se escuche claramente la voz de cada actor o actriz.

Cuando hacemos teatro, se nos plantea el reto de transmitir al público lo que queremos (Álvaro16). Mi miedo es equivocarme y hacer el ridículo (Ainhoa14). Los nervios. Que te superen los nervios. Que te pongas muy nerviosa y te salga todo mal (Nerea12).

También es un riesgo revelar la propia vida personal durante la realización las actividades dramáticas, pues no hay que olvidar que estas siempre son proyectivas: Hay otro riesgo, que es el personal, el de contar las historias en primera persona (Marta 14).

Compromiso (COM), subcategoría con $4.96 \%$, alude a que el teatro requiere tomar parte de un proceso de trabajo disciplinado y comprometido. Los participantes lo entienden como:

Todos tenemos que estar pendientes de todos (Josemi19).

También lo ven como exigencia personal: 
Contigo mismo sobre todo y con el grupo (Josemi19). Con la obra en sí y con la directora (Soraya16). Y con el público, porque si lo haces mal, el público tampoco lo va a pasar bien (Carla17).

Los participantes tienen conciencia de que el compromiso implica esfuerzo continuado:

Para hacer algo hace falta continuidad (Pau18).

Así metafóricamente lo declara uno de los participantes:

No hay arco iris sin lluvia (Rubén16).

\section{Autoconciencia emocional}

Se refiere a la oportunidad que el teatro ofrece para expresar sentimientos y pensamientos en un contexto seguro. La subcategoría Expresión de sentimientos (EXS), con el 6.27\%, reúne las unidades temáticas que referidas a la coyuntura que proporciona hacer teatro y actividades dramáticas para expresar percepciones, emociones y puntos de vista.

Yo en teatro, puedo expresar mejor mis sentimientos porque conozco más a la gente. [...] Me cuesta muchísimo menos expresar mis sentimientos con ellos, que luego fuera, con la gente de mi instituto. Para mí es mucho más fácil hacerlo dentro de las clases de teatro (Frida15).

Los participantes también declaran que son capaces de reconocer los sentimientos de las otras personas. Lo que conlleva el desarrollo de la empatía.

Puedes sentir cómo otras personas se sienten (Laura14). Te ayuda a que pienses en los demás y veas cómo se sienten (Soraya16).

La subcategoría Seguridad (SEG) hace referencia a la percepción de que el grupo de teatro es un contexto seguro. A pesar de su bajo porcentaje (el $3.75 \%$ de las unidades temáticas) representa la centralidad en el mapa de las relaciones semánticas (ver figura 1). En este sentido, la expresión de sentimientos es 
una propiedad de la seguridad. Además, se asocia con la opinión que se tiene sobre el teatro, con la conciencia de apoyo, con el compromiso, con las relaciones de amistad y con los cambios personales percibidos. Es la causa de producir transferencias de lo aprendido y está en contradicción con los retos percibidos.

FIGURA 1. Red de relaciones semánticas de las subcategorías

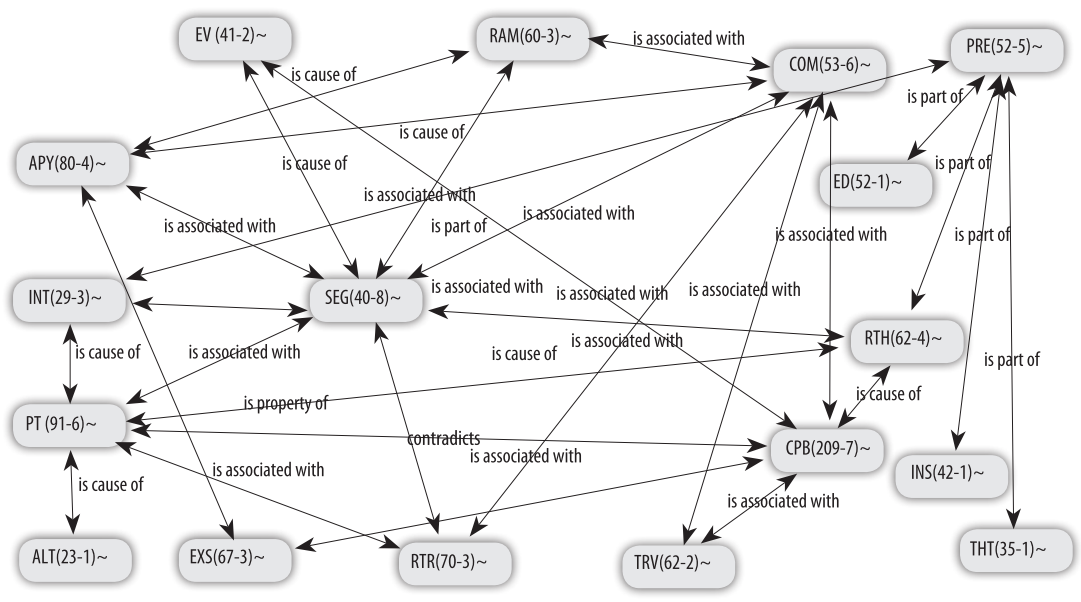

Fuente. Elaboración propia

Este sentimiento de seguridad de protección y confianza lo manifiestan los participantes con declaraciones como:

Te da mucha seguridad (Ainhoa14). A mí, por ejemplo, el teatro me ha hecho ganar seguridad (Frida15).

\section{Relaciones personales}

Aquí se agrupan las unidades temáticas relacionadas con el sentimiento de apoyo, percibido en el grupo de teatro, y con los lazos de amistad que en él se establecen.

La subcategoría Relaciones de amistad (RAM), con 5.62\%, se refiere a que el teatro es considerado como un medio que permite establecer relaciones distintas a las establecidas con las personas con que uno se codea en el día a día. 
Somos una piña, somos una familia, estamos todos juntos (Sonia16). Ya no somos unidades individuales sino que cuando hacemos teatro y después, cuando nos juntamos todos, yo siento que somos como un grupo, somos uno (Álvaro16).

Este es mi primer ańo en el instituto y gracias al teatro he podido conocer a mucha más gente (Nerea12). Lo importante es que seamos un grupo. No que seamos "un grupo de teatro", sino un grupo de verdad (Frida 15).

La subcategoría Apoyo (APY) (7.49\%) se refiere a la autoconciencia de sentirse protegido.

Si falla uno, todos se preocupan por él. Es como un equipo en el que todos damos y aportamos cosas (Alejandro16). Nos mezclamos varios cursos. Eso hace [...] que los mayores ayuden a los más pequeños y que los mayores siempre tengan como una pequeña responsabilidad (Nerea12).

\section{Discusión}

Los resultados invitan a realizar una serie de consideraciones en relación con los beneficios percibidos de la vivencia de la experiencia teatral.

\section{Primera hipótesis}

Según los resultados obtenidos, hemos podido constatar que los adolescentes de la muestra resaltan la utilidad que tiene para su desarrollo personal, ya sea hacer teatro o participar en actividades dramáticas en general. Así se confirma en la subcategoría Cambio personal de la categoría Beneficios. Sus resultados hacen referencia a autoestima, mayor imaginación, superación de miedos y de timidez al actuar, al hablar o hacer alguna actividad ante los demás y mejora la expresión oral, la escucha, espontaneidad, respeto a los demás, mayor responsabilidad y ser más abiertos y comunicativos. Estos concuerdan con la investigación de Eccles et al. (2003) que encontraron mayores habilidades de comunicación y, en general, más habilidades sociales en los sujetos que participaban en actividades extraescolares. Por otra parte, los par- 
ticipantes afirman que el teatro les ha ayudado y enseñado cosas diversas como a utilizar la imaginación, escuchar, superar miedos, ser más espontáneos, etcétera.

Por todo ello, podemos concluir que los adolescentes de la muestra valoran positivamente la experiencia de hacer teatro pues declaran que les aporta efectos beneficiosos para sus vidas.

\section{Segunda hipótesis}

Respecto a la segunda hipótesis, encontramos que los resultados muestran que haciendo teatro se incrementan, como mínimo, cuatro de las cinco $\mathrm{C}$ del programa para el desarrollo positivo juvenil implementado por Lerner et al. (2011). A saber:

1. Competencia. El teatro ayuda a desarrollar una visión más positiva de las propias acciones.

2. Conexión. Para expresar esta dimensión los sujetos de la muestra utilizan metáforas como "familia" o "piña". Al ser el teatro una actividad grupal, desarrolla las habilidades sociales y comunicativas. Por ello los participantes declaran estar unidos y pertenecer al grupo.

3. Cuidado. Los sujetos de la muestra manifiestan que hacer teatro les ayuda a empatizar, a pensar en los demás y a percibir cómo sienten los otros.

4. Confianza. Entre los cambios personales que los participantes declaran haber experimentado está la superación de la vergüenza, que hace que se sientan más abiertos y comunicativos.

Al repasar cada una de las capacidades recogidas en la segunda hipótesis encontramos los siguientes resultados.

a) Expresión y manejo de las propias emociones y las de otras personas. Las declaraciones recogidas en la subcategoría Expresión de sentimiento, de la categoría Autoconciencia emocional hacen referencia genérica a la facilidad para expresar sentimientos y poder apreciar cómo se sienten los otros. Al entrar en el espacio seguro, que el teatro representa, permite expresar impresiones y pensamientos, tomar conciencia de ellos y no tener miedo de exponerlos ante los demás. 
b) Compromiso. Los participantes entienden el compromiso como: "todos tenemos que estar pendientes de todos" porque "actuar es compromiso". Y este ha de ser "con uno mismo sobre todo y con el grupo", "con la obra en sî", con "la directora” y "con el público". Los participantes lo consideran como exigencia personal.

c) Sentido de la pertenencia y vinculación. Los participantes declaran haber establecido relaciones con los compañeros del grupo de teatro distintas a las mantenidas con otros iguales. Ello puede explicarse desde el modelo de desarrollo de las artes escénicas en los jóvenes de Beare y Belliveau (2007). La mayor parte de los sujetos de la muestra se encuentran en una de las tres primeras etapas (inclusion, control, intimacy). Los participantes en actividades teatrales tienen la necesidad de integrarse en el grupo y sentirse protegidos. Los iguales son una fuente importante de apoyo emocional, afecto y solidaridad. La interacción con los iguales se convierte en un escenario para probar "quien soy" y "quien podría ser" y desarrollar la propia autonomía e independencia (Youngblade et al. 2007).

Participar en actividades teatrales favorece la autoconciencia de sentirse apoyado en un ambiente de seguridad. Pertenecer a un grupo de teatro consolidado hace que los adolecentes se sientan acogidos por sus pares.

d) Evasión. La especial experiencia de "salir de tu mundo" y "vivir a través de otros yoes" que el hacer teatro supone es la razón por la que los participantes declaran que es un espacio de evasión donde olvidar y escapar por unas horas de los problemas personales. El contexto de liminalidad (Turner, 2001) y la teoría de la metaxis (Boal, 2004) explican este poder liberador del teatro que encontramos en las declaraciones de los participantes recogidas en la subcategoría Evasión de la categoría Beneficios.

e) Habilidades sociales. Las declaraciones referidas a las habilidades sociales y comunicativas se agrupan en la subcategoría Relaciones de amistad de la categoría Relaciones personales. Los participantes manifiestan haber establecido estrechos la- 
zos de amistad y sienten el grupo de teatro como una familia. Se encuentran en la etapa intimacy de Beare y Belliveau (2007) y en el área de intervención connection de Lerner et al. (20119 Los resultados obtenidos son acordes con las conclusiones de Daykin et al. (2008) para quienes existen suficientes evidencias para afirmar que las prácticas dramáticas tienen efectos positivos sobre las habilidades sociales. Esto se explica porque el teatro es una actividad grupal y por ello desarrolla determinadas capacidades que guardan relación con la necesidad de pertinencia de los adolescentes.

f) Respecto a las habilidades comunicativas, hay referencias en las subcategorías Beneficios personales de la categorías Beneficios. El contenido de tales versa sobre la expresión oral (tener más vocabulario, expresarse mejor, aprender a hablar en público, transmitir claramente un mensaje), aprender a escuchar, aprender a respetar el punto de vista de los otros y ser más abierto con la gente. La relación del teatro y las capacidades comunicativas están suficientemente confirmadas en Hughes y Wilson (2004).

g) Transferencia. De sus opiniones podemos deducir que tomar parte en actividades teatrales de alguna forma proporciona destrezas que luego pueden ser transferidas a otras situaciones y momentos de la vida. Esta capacidad se corresponde con áreas de intervención contribution de Lerner et al. (2011).

Sería necesario utilizar instrumentos más precisos que los grupos foco para poder comprobar con más precisión los efectos del teatro en cada una de las capacidades enumeradas en este apartado.

\section{Tercera hipótesis}

Constatamos que los sujetos de la muestra declaran tener conciencia de que el teatro es una herramienta y una oportunidad para plantearse su identidad, conocerse mejor y crecer como persona. Las expresiones sobre la identidad aparecen fundamentalmente en la categoría Beneficios y la expresan con frases tales como: "crecer como persona", "ser mejor persona", "ser uno mismo", "ser lo que uno quiere ser", "encontrarse con uno mismo". Enun- 
ciados que están en sintonía con teorizaciones sobre la identidad de Erikson (1983) y Kroeger (2004). Las actividades dramáticas dan a los jóvenes la oportunidad de explorar otros "posibles yoes" lo que les ayudará en el proceso de construcción su propia identidad (Halverson, 2010), pues hacer teatro es como salir de tu mundo y poder jugar con diferentes identidades. El adoptar distintos roles es especialmente importante para el proceso y el desempeño creativo (Hughes y Wilson, 2004). En el espacio sin sanción que es el teatro, los participantes pueden probar experiencias sin sus consecuencias concretas, bajo la influencia de sus pares en un ambiente seguro donde discutir y explorar los sentimientos personales y ayudar a desarrollar una identidad positiva y saludable.

Por otra parte, el teatro tiene la capacidad de crear un contexto que facilita la liminalidad (Boal, 2004 y Turner, 2001). Y así, a su manera, lo expresan los participantes cuando se les pregunta qué es el teatro para ellos: "ponerse en la piel de otra persona", "meterse en su vida", "olvidar uno quien es", etcétera.

Respecto a la autoestima hay numerosas unidades de contenido referidas al incremento de la confianza, que supone una mejora en la autoestima. Encontramos declaraciones como: el teatro me ha ayudado a subir la autoestima, tenía muy poca autoestima o ahora tengo más confianza en mí mismo. Estos resultados son acordes con lo que afirman Broh (2002) y Barquero y Vargas (2009) y se corresponden con desarrollo de la capacidad confidence (Lerner et al., 2011). El refuerzo de la autoestima es una de los beneficios de las actividades teatrales aceptado por la mayoría de los autores.

Para cuantificar las unidades temáticas referidas a la identidad y a la autonomía hubiera sido necesario haber establecido dos subcategorías más en Beneficios. De este modo hubiéramos podido comprobar el peso de estas dimensiones en la relación entre actividades teatrales y desarrollo positivo.

En cuanto al teatro como arte, constatamos que las declaraciones, recogidas en la categoría Pensamiento sobre el teatro, se corresponde con los resultados de la investigación de Sires y Bayona (2006) respecto a los géneros preferidos por los adolescentes (comedia, musical, tragedia) y a las características de los espectáculos (calidad, dinámicos, impactantes y con formato diferente, 
humor inteligente, etc.) Y con los de Needlans (2008) sobre los rasgos de un teatro juvenil (contexto local, relevancia, innovador y experimental y basado en el grupo). Y respecto a la iniciación al teatro, los resultados de esta investigación son acordes con McCarthy, K. y Jinnet, K. (2001) sobre la influencia de los pares, la familia, la escuela y las experiencias tempranas para llegar a ser espectadores de teatro o participar en actividades dramáticas. La influencia social y el aprendizaje vicario son las razones de más peso a la hora de iniciarse en la práctica del teatro.

\section{Limitaciones}

Las declaraciones de los sujetos de la presente investigación son en ocasiones muy contundentes y adolecen de matices. Consideramos que se trata de un rasgo propio de su edad, pero puede ser una de las limitaciones del presente estudio.

En este sentido, ¿el hecho de que muchos de los participantes hayan protagonizado o visualizado un espectáculo poco antes de participar en los grupos de discusión puede afectar en la intensidad de sus declaraciones y suponer un sesgo positivo?, ¿hasta qué punto los resultados de la investigación son una confirmación de la teoría y en qué medida pueden ser vistos como una sólida "evidencia" sobre el impacto que tiene la participación en los jóvenes en el teatro? Estas preguntas requieren de otras investigaciones que ayuden a encontrar respuestas satisfactorias que complementarían los resultados aquí expuestos. Otra limitación reside en que los resultados están basados en datos generados por participantes comprometidos en grupos de teatro, y no se han contrastado con un grupo control. De aquí la necesidad de seguir investigando esta temática con otros participantes y otros diseños de investigación y el planteamiento de diseños longitudinales con medidas de cambio intraindividual e interindividual en distintas capacidades.

Si la práctica teatral parece tener influencias beneficiosas sobre el desarrollo psicológico de los jóvenes, es necesario desarrollar metodologías para valorar su intensidad, comprender mejor la naturaleza de dicha influencia, con el fin de diseñar programas de intervención encaminados a ese fin. 


\section{Referencias bibliográficas}

Alsinet, C, Pérez, R. y Agullo, M. "Adolescentes y percepciones del riesgo", en Revista de Estudios sobre juventud, núm. 18, 2003, pp. 90-101.

Beare, D. y Belliveau, G. "Theatre for Positive Youth Development: A Development Model for Collaborative Playcreating", en Applied Theatre Research, núm. 8, 2007. Disponible en https://www.griffith.edu.au/_data/assets/ pdf_file/0008/52892/04-beare-belliveau-final.pdf

Benson, P. L., Scales, P. C., Hamilton, S. F. y Sesman, A., J. R. Positive youth development: Theory, research and applications, en R. M. Lerner (Ed.), Theoretical models of human development. Volume 1 of Handbook of Child Psychology (2006, 6a ed., pp. 894-941). Hoboken, N.J., Wiley.

Benson, P. L., Mannes, M., Pittman, K. y Ferber, T. "Youth development, developmental assets and public policy", en R. Lerner y L. Steinberg (Eds.), Handbook of adolescent psychology (2004, 2a ed., pp. 781-814). Nueva York, John Wiley.

Berk, L. E. Development throug the Lifespan (3er. ed.), Slade River, N. J. Pearson Education, 2004.

Boal, A. El arco iris del deseo, Barcelona, Alba, 2004.

Broh, B. A. "Linking extracurricular programming to academic achievement: Who benefits and why?", en Sociology of Education, 75, 2002, pp. 69-96.

Daykin, N., Orme, J., Evans, D., Salmon, D., McEachran, D. and Brain, S. "The impact of participation in performing arts on adolescent health and behaviour: a systematic review of the literatura", en Journal of Health Psychology, 13(2), 2008, pp. 251-264.

Davis, S. "Perezhivanie and experience of drama, metaxis and meaning making", en Drama Australia Journal, 39 (1), 2015, pp. 63-75.

Dutton, S. E. "Urban Youth Development-Broadway Style: Using Theatre and Group Work as Vehicles for Positive Youth Development", en Social Work with Groups, 23 (4), 2001, pp. 39-58. 
Eccles, J. S., Barber, B. L., Stone, M. y Hunt, J. (2003). "Extracurricular activities and adolescent development", en Journal of Social Issues, 59, 2003, pp. 865-889.

Erikson, E. H. Infancia y sociedad, Buenos Aires, Horme-Paidós, 1983.

Estévez, E. Ferrer, B. y Musitu, G. "La autoestima en adolescentes agresores y víctimas en la escuela: La perspectiva multidimensional", en Intervención Psicosocial, 15 (2), 2006, pp. 223-232.

Fernández, F. "El análisis de contenido como ayuda metodológica para la investigación”, en Ciencias Sociales, II (96), 2002, pp. 35-53. Disponible en http://www.redalyc.org/ articulo.oa?id=1530960

Garaygordobil, A. y Durá, A. "Relaciones del autoconcepto y la autoestima con la sociabilidad, estabilidad emocional y responsabilidad en adolescentes de 14 a 17 años", en Análisis y Modificación de Conducta, 32, (141), 2006. Disponible en http://www.uhu.es/publicaciones/ojs/index. php/amc/article/viewFile/2132/2107

Gardner, M., Roth, J. y Brooks-Gunn, J. "Adolescents' participation in organized activities and developmental success two and eight years after high school: Do sponsorship, duration, and intensity matter?", en Developmental Psychology, 44, 2008, pp. 814-830.

Halverson, E. Artistic production processes as venues for positive youth development. WCER, paper n. 2010-2, Wisconsin: Center for Education Research. Disponible en http:// wcer-web.ad.education.wisc.edu/docs/working-papers/ Working_Paper_No_2010_02.pdf

Hughes, J. y Wilson, K. "Playing a part: the impact of youth theatre on young people's personal and social development", en Research in Drama Education, 9, (1), 2004, pp. 57-72.

Jiménez, T., Musitu, G. y Murgui, S. "Familia, apoyo social y conducta delictiva en la adolescencia: efectos directos y mediadores", en Anuario de Psicología, 36 (2), 2005, pp. 181-195. 
Johnson, L. y O'Neill, C. (Ed.). Collected Writing in Drama Education, Dorothy Heathcote, Evanston, I. L., Northwestern University Press, 1991.

Kim, A. J. et al. "Neurodiversity on the Stage: The Effects of Inclusive Theatre on Youth with Autism", en International Journal of Education and Social Science, 2, (9), 2015, pp. 27-39.

Kroeger, J. Identity in Adolescence. The balance between self and other (3er. ed.), New York, Rouledge, 2004.

Laferrière, G. y Motos, T. Palabras para la acción: Términos de teatro en la educación y la intervención sociocultural, Ciudad Real, Naque, 2003.

Larson, R., Jarrett, R., Hansen, D., Pearce, N., Sullivan, P., Walker, K., Watkins, N. y Wood, D. "Organized youth activities as contexts of positive development", en A. Linley y S. Joseph (Eds.), Positive psychology in practice, New York, Wiley, 2004, pp. 540-560.

Lerner, R. M., Lerner, J. V. and Collegues. The Positive Development of Touth. Report of the Findings from the Seven Years of the 4-H Study of Positive Youth Development, Institute for Applied Research in Youth Development, Tufts University, 2011.

Lerner, R. M. y Steinberg (Eds.). Handbook of Adolescence Psycology, Hoboken, N. Y., John Wiley and Sons, 2011.

Marcia, J. E. "Identity in adolescence", en J. Adelson (Ed.), Handbook of Adolescent Psychology, Nueva York, Wiley, 1980, pp. 17-39.

McCarthy, K. y Jinnet, K. A New Framework for Building. Participation in the Arts, Santa Mónica, RAND, 2001. Disponible en http://www.rand.org/content/dam/rand/pubs/ monograph_reports/2005/MR1323.pdf

Navarro, R. Valor pedagógico de la Dramatización. Su importancia en la Formación Inicial del Profesorado (Tesis doctoral no publicada), Universidad de Sevilla, 2005.

O'Neill, C. Drama Words: A Framework for Process Drama, Portsmouth, N. H. Heinemann, 1995.

Neelands, J., Baldwin, P. and Fleming, K. Teaching Literacy through Drama: Creative Approaches, London, Routledge Falmer, 2003. 
Needlands, J. Essentially Youth Theatre. NAYAD Starting The Debate Seminar May10th, 2008. Disponible en http:// nayad.ie./files/jNeedlandsadress.pdf

Oliva, A., Ríos, M., Antolín, L., Parra, A., Hernando, A. y Pertegal, M. A. "Más allá del déficit: construyendo un modelo de desarrollo positivo adolescente", en Infancia y Aprendizaje, 2010, 33 (2). Disponible en https://idus.us.es/xmlui/ bitstream/handle/11441/32595/M\%E1s\%20all\%E1\%20 del $\% 20 \mathrm{~d} \%$ E9ficit $\% 20$ postprint.pdf?sequence $=1$

Organización Mundial de la Salud. La salud de los jóvenes: un desafio para la sociedad. Serie informes técnicos, 731, Ginebra, OMS, 1986.

Persson, A., Kerr, M. y Stattin, H. "Staying in or moving away from structured activities: explanations involving parents and peers", en Developmental Psychology, 43, 2007, pp. 197-207.

Schechner, R. Between Theater and Anthropology, University of Pennsylvania Press, 1985.

Sires, T. y Bayona, M. "Estudi qualitatiu dels joves adolescents i els teatres de la comarca del Vall's Oriental", en Les barreres d'acces, Barcelona, Diputació de Barcelona, 2006.

Stassen, K. Psicología del Desarrollo: Infancia y Adolescencia (7a ed.), Madrid, Editorial Médica Panamericana, 2007.

Taylor, S. J. y Bogdan, R. Introducción a los métodos cualitativos de investigación: La búsqueda de significados, México, Paidós, 1986.

Turner, V. "From Ritual to Theatre: The Human Seriousness of Play", en Performing Arts Journal Publication, Nueva York, 2001.

Youngblade, L. M., Theokas, C., Schulenberg, J., Curry, L., Huang, I-C., Novack, M. Risk and promotive factors in families, schools, and communities: A contextual model of positive youth development in adolescence, en Pediatrics, 119 Suppl., S47-S53, 2007.

Zacares, J., Iborra A. Tomás, J. M. y Serra, E. "El desarrollo de la identidad en la adolescencia y adultez emergente: Una comparación de la identidad global frente a la identidad en dominios específicos”, en Anales de Psicología, 25, (2), 2009, pp. 316-329. 